\title{
The String Stability of a Trajectory-Based Interval Management Algorithm in the Midterm Airspace
}

\author{
Kurt A. Swieringa* \\ NASA Langley Research Center, Hampton, VA, 23681, United States
}

\begin{abstract}
NASA's first Air Traffic Management (ATM) Technology Demonstration (ATD-1) was created to facilitate the transition of mature ATM technologies from the laboratory to operational use. The technologies selected for demonstration are the Traffic Management Advisor with Terminal Metering (TMA-TM), which provides precise time-based scheduling in the terminal airspace; Controller Managed Spacing (CMS), which provides terminal controllers with decision support tools enabling precise schedule conformance; and Interval Management (IM), which consists of flight deck automation that enables aircraft to achieve or maintain a precise spacing interval behind a target aircraft. As the percentage of IM equipped aircraft increases, controllers may provide IM clearances to sequences, or strings, of IM-equipped aircraft. It is important for these strings to maintain stable performance. This paper describes an analytic analysis of the string stability of the latest version of NASA's IM algorithm and a fast-time simulation designed to characterize the string performance of the IM algorithm. The analytic analysis showed that the spacing algorithm has stable poles, indicating that a spacing error perturbation will be reduced as a function of string position. The fast-time simulation investigated IM operations at two airports using constraints associated with the midterm airspace, including limited information of the target aircraft's intended speed profile and limited information of the wind forecast on the target aircraft's route. The results of the fast-time simulation demonstrated that the performance of the spacing algorithm is acceptable for strings of moderate length; however, there is some degradation in IM performance as a function of string position.
\end{abstract}

\section{Nomenclature}

ANOVA Analysis of Variance

ASTAR-11 Airborne Spacing for Terminal Arrival Routes version 11 (previous version)

ASTAR-12 Airborne Spacing for Terminal Arrival Routes version 12 (current version)

ATD-1 ATM Technology Demonstration

ATM Air Traffic Management

$B A D A \quad$ Base of Aircraft Data

CMS Controller Managed Spacing

ETA Estimated Time of Arrival

FAA Federal Aviation Administration

IM Interval Management

NASA National Aeronautics and Space Administration

$O P D \quad$ Optimized Profile Descent

STAR Standard Terminal Arrival Route

TMA-TM Traffic Management Advisor with Terminal Metering

${ }^{*}$ Research Engineer, Crew Systems and Aviation Operations Branch, NASA LaRC, MS 152., SS 


\section{Introduction}

VER the next twenty years, the Federal Aviation Administration (FAA) is predicting a substantial increase

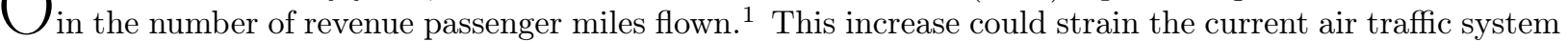
if improvements are not made. To help increase the efficiency of arrivals, new procedures, such as Optimized Profile Descents (OPDs), have been implemented at a number of sites. However, these procedures are often not used during periods of peak traffic due to the lack of precision scheduling and spacing tools, ${ }^{2}$ resulting in sub-optimal arrival operations. The National Aeronautics and Space Administration (NASA) has been developing technologies that are expected to increase the use of efficient arrival procedures during periods of peak traffic.

The goal of NASA's first Air Traffic Management (ATM) Technology Demonstration (ATD-1) is to accelerate the implementation of mature NASA technologies, enabling aircraft to use speed control to remain close to their OPDs during periods of peak traffic. ${ }^{3}$ Three NASA technologies were selected to be integrated together to achieve this goal: the Traffic Management Advisor with Terminal Metering (TMA-TM) ${ }^{4,5}$ which provides precise time-based scheduling in the terminal airspace; Controller Managed Spacing (CMS), ${ }^{6,7}$ which provides terminal controllers with decision support tools that enable precise schedule conformance; and Interval Management (IM), which consists of flight deck automation that enables aircraft to achieve or maintain a precise spacing interval behind the preceding aircraft. ${ }^{8,9}$ These three technologies extend precision scheduling into the terminal area and provide pilots and controllers with control mechanisms that improve schedule conformance and in-trail spacing precision.

The IM algorithm that NASA has been developing is a trajectory-based IM algorithm called the Airborne Spacing for Terminal Arrival Routes (ASTAR) algorithm. ${ }^{10,11}$ The ASTAR algorithm uses the intended trajectories of both the IM and target aircraft to calculate their Estimated Times of Arrival (ETAs). The ETAs are used to calculate a spacing error and the speed control required to null the spacing error by an achieve-by point. Previous versions of the ASTAR algorithm have shown good performance in several batch simulations and human-in-the-loop simulations. ${ }^{12-18}$

The ATD-1 flight demonstration will occur in a midterm airspace environment where future communication infrastructure, such as controller-pilot data link communication, is not available. To compensate for the lack of accurate target aircraft trajectory intent information, NASA's ASTAR spacing algorithm uses constraints from published Standard Terminal Arrival Routes (STARs) to compute the ETAs of both the IM and target aircraft. Discrepancies between the published speed profile and delayed speed profiles computed by TMA-TM cause the speeds flown by the target aircraft to deviate from the published speeds expected by the spacing algorithm. Additionally, the IM aircraft is not expected to have access to the target aircraft's wind forecast for the ATD-1 demonstration, increasing the uncertainty of the target aircraft's ETA calculations when the target aircraft is on a different route.

The latest version of the ASTAR algorithm, ASTAR-12, includes modifications to the speed control algorithm that improve compatibility with TMA-TM and CMS when a delayed target aircraft deviates from its expected groundspeed. A previous batch simulation examined pairs of aircraft, consisting of a single IM aircraft following a single target aircraft, conducting IM operations using the ASTAR-12 algorithm. ${ }^{19}$ The simulation found that the ASTAR-12 algorithm's delivery accuracy and precision were generally acceptable; however, the batch simulation did not examine the performance of the ASTAR-12 algorithm when there is a string of several IM aircraft following each other. This paper describes both the fundamental string stability of the ASTAR-12 spacing algorithm and a fast-time simulation designed to emulate strings of aircraft performing IM operations in the midterm national airspace system.

The first section of this paper provides an overview of previous IM research. The following section describes the fundamental string stability behavior of the ASTAR-12 algorithm using an analytic approach. The final two sections describe a fast-time simulation that investigated the performance of strings of IM aircraft using the ASTAR-12 spacing algorithm, assuming midterm airspace constraints.

\section{Background}

\section{A. Previous IM Algorithm Research}

IM research has been conducted since the 1970's. The early IM work focused on the development of IM algorithms, such as time history algorithms and constant time delay algorithms, that used state information

from the target aircraft to determine the spacing error. ${ }^{20}$ Within these algorithms, the spacing error is 
defined as the difference between a spacing goal assigned by air traffic control and the time elapsed between the time when the target aircraft passed the IM aircraft's current position and the time when the IM aircraft arrives at that position. Due to the definition of spacing error, these algorithms are limited to operations where the IM and target aircraft are in-trail.

More recently, trajectory-based IM algorithms have been developed. The NASA-developed Airborne Spacing for Terminal Arrival Routes (ASTAR) is a trajectory-based IM algorithm that was designed to be used in a NextGen environment where detailed information of the target aircraft's intended trajectory is available to the IM aircraft. ${ }^{11,12}$ The ASTAR algorithm uses predicted trajectories of the IM aircraft and target aircraft to calculate a time-to-go for each aircraft to a common achieve-by point. The spacing error is then calculated by comparing the difference between the time-to-go of the IM and target aircraft with a spacing goal assigned by air traffic control. Several batch simulations and human-in-the-loop simulations have demonstrated that the ASTAR algorithm is able to precisely deliver aircraft to the achieve-by point and that the speed produced by the algorithm are generally acceptable to pilots. ${ }^{8,9,17,21}$ Each of these human-in-the-loop simulations assumed an advanced airspace environment with controller-pilot datalink communications used to transmit IM clearances to the flight deck, and that the IM aircraft will have access to detailed information of the target aircraft's intended trajectory.

When the ATD-1 project began, the focus of IM research at NASA switched from a future environment that included controller-pilot datalink communications to the use of IM in the midterm airspace environment. Since controller-pilot data link communication is not expected to be available in the midterm national airspace system, IM clearances are provided using voice communications and the intended trajectories of the IM and target aircraft are assumed to be published Standard Terminal Arrival Routes (STARs). ${ }^{3,15,16,22}$ In preparation for the ATD-1 flight demonstration, simulations were also conducted to examine the integration of IM with TMA-TM and CMS (also known as Terminal Sequencing and Spacing).

The previous version of the ASTAR algorithm, ASTAR-11, did not perform well when used with TMATM and the CMS tools. TMA-TM uses the predicted trajectory of each aircraft along their projected OPDs to compute their ETAs to a series of scheduling waypoints. If there is a conflict at one of the scheduling waypoints, TMA-TM often delays aircraft. With the advent of flex scheduling, aircraft can also be advanced in certain circumstances. Since ASTAR used the published STARs as the estimate of the target aircraft's intended trajectory, the speeds expected by ASTAR and TMA-TM do not always match. The ASTAR-11 algorithm was not designed to compensate for large speed differences between the target aircraft's actual speed and the published speeds that were used by ASTAR-11 to predict the target aircraft's ETA. The result was that the ASTAR-11 algorithm exhibited a large steady state error and undesirable closure rates with the target aircraft when the target aircraft was absorbing delay. ${ }^{19}$

NASA's ASTAR algorithm was updated to mitigate the previously described problems and improve compatibility with TMA-TM and CMS. ${ }^{19}$ The main modification was a groundspeed term that was added to the ASTAR algorithm to compensate for discrepancies between the target aircraft's actual speeds and published speeds. The groundspeed term essentially enables the IM aircraft to match the target aircraft's speed deviation and then correct for the spacing error using the proportional control term. The groundspeed term also prevents steady-state errors from occurring when the the target aircraft is not flying its expected speed, reducing undesirable closure rates between the IM and target aircraft. Both human-in-the-loop simulations and batch simulations investigated the performance of the ASTAR-12 algorithm and its acceptability to pilots and controllers. However, these studies did not focus on the string behavior of the ASTAR-12 spacing algorithm.

\section{B. String Stability Research}

When there are a number of IM-equipped aircraft arriving into an airport, it is possible for several IM aircraft to follow each other, creating a string of IM aircraft. A spacing algorithm is considered to be string stable if the spacing error does not grow as a function of string position, ensuring that small perturbations early in the string do not develop into unacceptable behavior later in the string.

Figure 1 shows an example of a string of IM aircraft. The aircraft are depicted as being in-trail; however, when a trajectory-based algorithm is used, aircraft within a string can either be on in-trail or on different routes. Throughout the analytical analysis described in section III, increasing values of $i$ refer to moving backwards in the string and decreasing values refer to moving forward in the string.

There is a substantial amount of research that has investigated the string stability of various IM algorithms. Weitz and Hurtado examined the string stability of several IM algorithms using both an analytic 


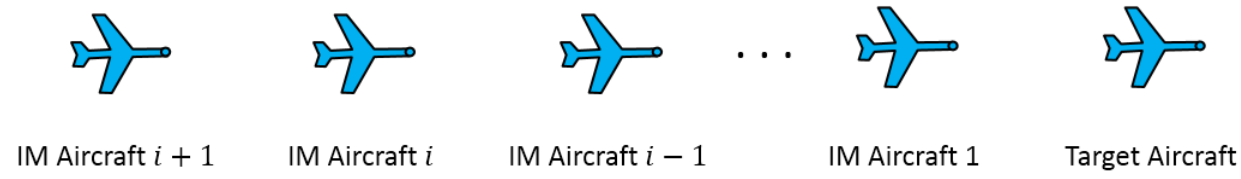

Figure 1. Example of a string of IM aircraft

approach and a fast-time simulation. ${ }^{23}$ One of the algorithms they examined was a time-to-go algorithm that is similar to a previous version of the ASTAR spacing algorithm (ASTAR-11). They found that the time-to-go algorithm was weakly string stable. Krishnamurthy et al. conducted a fast-time simulation that examined strings consisting of $100 \mathrm{IM}$ aircraft. ${ }^{24}$ The results of the fast-time simulation did not reveal any string instabilities.

The string stability of various spacing algorithms have also been validated in previous human-in-the-loop simulations. In 2005, Barmore et al. described a human-in-the-loop simulation that included strings of nine IM aircraft. The results of the simulation did not reveal any string instabilities. ${ }^{13}$ Baxley et al. conducted a human-in-the loop simulation that examined a previous version of NASA's ASTAR spacing algorithm. ${ }^{18,21}$ During the simulation, strings of 23 IM aircraft, including six pilot flown aircraft, were examined for string instabilities; no string instabilities were found.

\section{ASTAR-12 Spacing Algorithm Description}

ASTAR-12 is a trajectory-based spacing algorithm designed to support IM operations both when the IM and target aircraft are in-trail and when they are on merging routes. The spacing error is calculated using the time-to-go of the IM and target aircraft along their predicted 4D-trajectories. In the future, it will be possible for either the target aircraft's trajectory or estimated time of arrival at the achieve-by point to be communicated directly to the IM aircraft. However, this information is not available in the midterm airspace environment. To compensate for the lack of 4D-trajectory information, published Standard Terminal Arrival Routes (STARs) are used to predict both the IM and target aircraft's 4D-trajectories. The time-to-go for each aircraft is simply the difference between their ETA at the achieve-by point and the current time. The spacing error is then defined as the difference between IM aircraft's time-to-go $\left(T T G_{i}\right)$ to the achieve-by point and the target aircraft's time-to-go to the achieve-by point $\left(T T G_{i-1}\right)$ minus the spacing goal assigned by air traffic control $(\Delta)$.

$$
e_{i}(t)=T T G_{i}(t)-T T G_{i-1}(t)-\Delta
$$

The amount of speed control required to null the spacing error is computed using a proportional controller with a groundspeed term added to compensate for differences between the target aircraft's predicted groundspeed and actual groundspeed. The speed control is added to the IM aircraft's nominal speed to generate an IM commanded speed $\left(v_{c m d_{i}}\right)$.

$$
v_{c m d_{i}}(t)=v_{n o m_{i}}(t)+k_{p} e_{i}(t)+k_{G S}\left(v_{\text {der }_{i-1}}(t)-v_{\text {nom }_{i-1}}(t)\right)
$$

Here $v_{n o m_{i}}$ is the nominal 4D-trajectory airspeed of the IM aircraft; $k_{p}$ is the proportional gain, $e_{i}(t)$ is the spacing error; $k_{G S}$ is the gain for the groundspeed term; $v_{d e r_{i-1}}$ is the airspeed derived from the target aircraft's groundspeed, wind forecast, and altitude; and $v_{n o m_{i-1}}$ is the nominal 4D-trajectory airspeed of the target aircraft.

The IM avionics used in the ATD-1 flight demonstration will likely be a retrofit implementation that is not connected with the aircraft's autoflight system, requiring the flight crew to monitor for IM speed changes and enter them into the aircraft's mode control panel speed window to close the control loop. To reduce the number of speed changes that the flight crew is required to respond to, speed commands are discretized into either five or ten knot increments prior to being displayed to the flight crew.

Since the ASTAR-12 algorithm is designed to support an achieve-by IM operation, the ideal behavior is to smoothly null the spacing error between the point where the IM operation begins and the achieve-by point. Gain scheduling is implemented to ensure that the appropriate amount of speed control is used throughout the arrival. The proportional gain ranges from a value of 0.375 when the IM aircraft is far from the achieveby point to 1.5 when the IM aircraft is within $10 \mathrm{nmi}$ of the achieve-by point. The groundspeed gain is 
set to one until the IM aircraft is $40 \mathrm{nmi}$ from the achieve-by point. Between $40 \mathrm{nmi}$ and $20 \mathrm{nmi}$ from the achieve-by point, the groundspeed gain is linearly decreased to zero, and remains at zero until the achieve-by point. Additionally, the groundspeed gain is set to zero whenever the target aircraft's groundspeed is faster than expected. This is done to reduce the number of instances where ASTAR-12 commands a speed increase during an arrival and to increase conformance with the controller tools, which primarily resolve conflicts at meter points during high density operations by delaying aircraft.

Additional filtering is applied to the target aircraft's groundspeed differential to prevent undesirable speed changes from occurring. The target aircraft's groundspeed is filtered using a first order low pass filter to remove high frequency variability. The time constant of the filter changes from 60 seconds when the IM aircraft is more than $35 \mathrm{nmi}$ from the achieve-by point to zero when the IM aircraft is at the achieve-by point.

\section{Analytic Analysis of String Stability}

Long strings of IM aircraft could exhibit unstable string behavior if the control algorithm is not designed properly. This section describes an analytic analysis of the string stability of the ASTAR-12 algorithm. The first step of the analysis is to derive a transfer function for the error propagation through a string of IM aircraft. After the transfer function is derived, its poles can be calculated and used to determine if the error propagation transfer function is asymptotically stable. As is often the case with analytical analyses, several simplifying assumptions are made within this analysis:

- The time delay associated with various filters in ASTAR is not modeled;

- The time it takes the flight crew to recognize a speed command and enter it into their aircraft is not modeled;

- The conversion from airspeed to groundspeed is not modeled;

- The nominal speed of the IM and target aircraft are assumed to be both constant and equal; and

- Simplified aircraft dynamics are used.

The string stability of the ASTAR-12 control algorithm can be computed similarly to the analysis of the time-to-go algorithm described by Weitz and Hurtado. ${ }^{23}$ A simple one dimensional point mass is used to model the longitudinal aircraft dynamics. The aircraft's acceleration is assumed to be proportional to the difference between the IM commanded speed $\left(v_{c m d}\right)$ and the IM aircraft's current speed $\left(v_{i}\right)$, with a proportional gain equal to $k_{v}$. The variable $x$ refers to the distance traveled by the aircraft and the subscript $i$ refers to the $i^{t h}$ aircraft in the string.

$$
\begin{gathered}
\dot{x}_{i}(t)=v_{i}(t) \\
\dot{v}_{i}(t)=k_{v}\left(v_{c m d}(t)-v_{i}(t)\right)
\end{gathered}
$$

In a time-to-go algorithm, such as ASTAR-12, the spacing error is defined as the difference between the time-to-go of the IM aircraft and the time-to-go of the target aircraft minus the assigned spacing goal (equation 1). Assuming that the nominal profile speed is constant and ignoring the conversion from airspeed to groundspeed, the time-to-go is simply the along-path distance to the achieve-by point $\left(x_{A B P}-x(t)\right)$ divided by the nominal profile speed $\left(v_{n o m}\right)$. Thus, the spacing error and its first and second order time derivatives can be described using equations 6,7 , and 8 .

$$
\begin{aligned}
e_{i}(t) & =\frac{x_{A B P}-x_{i}(t)}{v_{\text {nom }}}-\frac{x_{A B P}-x_{i-1}(t)}{v_{\text {nom }}}-\Delta \\
& =\frac{x_{i-1}(t)-x_{i}(t)}{v_{\text {nom }}}-\Delta \\
\dot{e}_{i}(t) & =\frac{v_{i-1}(t)-v_{i}(t)}{v_{\text {nom }}} \\
\ddot{e}_{i}(t) & =\frac{\dot{v}_{i-1}(t)-\dot{v}_{i}(t)}{v_{\text {nom }}}
\end{aligned}
$$


The ASTAR-12 speed control law described in equation 2 is combined with the aircraft dynamics described in equation 4.

$$
\begin{aligned}
\dot{v}_{i}(t) & =k_{v}\left(v_{n o m}+k_{p} e_{i}(t)+k_{G S}\left(v_{i-1}(t)-v_{n o m}\right)-v_{i}(t)\right) \\
\dot{v}_{i-1}(t) & =k_{v}\left(v_{n o m}+k_{p} e_{i-1}(t)+k_{G S}\left(v_{i-2}(t)-v_{n o m}\right)-v_{i-1}(t)\right)
\end{aligned}
$$

Next, equations 9 and 10 are used in equation 8, and the resulting equation is rearranged and simplified into a differential equation describing the error propagation through the string of IM aircraft.

$$
\begin{aligned}
\ddot{e}_{i}(t)= & \frac{k_{v}\left(v_{n o m}+k_{p} e_{i-1}(t)+k_{G S}\left(v_{i-2}(t)-v_{n o m}\right)-v_{i-1}(t)\right)}{v_{n o m}} \\
& -\frac{k_{v}\left(v_{n o m}+k_{p} e_{i}(t)+k_{G S}\left(v_{i-1}(t)-v_{n o m}\right)-v_{i}(t)\right)}{v_{n o m}} \\
= & \frac{k_{v} v_{n o m}}{v_{n o m}}-\frac{k_{v} v_{n o m}}{v_{n o m}}+\frac{k_{v} k_{p} e_{i-1}(t)}{v_{\text {nom }}}-\frac{k_{v} k_{p} e_{i}(t)}{v_{n o m}}-\frac{k_{v} k_{G S} v_{n o m}}{v_{n o m}}+\frac{k_{v} k_{G S} v_{n o m}}{v_{n o m}} \\
& +k_{v} k_{G S}\left(\frac{v_{i-2}(t)-v_{i-1}(t)}{v_{n o m}}\right)-k_{v}\left(\frac{v_{i-1}(t)-v_{i}(t)}{v_{n o m}}\right) \\
= & \frac{k_{v} k_{p} e_{i-1}(t)}{v_{\text {nom }}}-\frac{k_{v} k_{p} e_{i}(t)}{v_{n o m}}+k_{v} k_{G S} \dot{e}_{i-1}(t)-k_{v} \dot{e}_{i}(t)
\end{aligned}
$$

The first and second terms in equation 12 cancel each other, the fifth and sixth terms cancel each other, and the seventh and the last term are equal to $\dot{e}_{i-1}(t)$ and $\dot{e}_{i}(t)$, respectively. After simplification, equation 13 is reduced to:

$$
\ddot{e}_{i}(t)+k_{v} \dot{e}_{i}(t)+\frac{k_{v} k_{p}}{v_{n o m}} e_{i}(t)=k_{v} k_{G S} \dot{e}_{i-1}(t)+\frac{k_{v} k_{p}}{v_{n o m}} e_{i-1}(t)
$$

A transfer function for spacing error is calculated by taking the Laplace transformation of equation 14 and rearranging.

$$
\frac{E_{i-1}}{E_{i}}=\frac{k_{v} k_{G S} s+\frac{k_{v} k_{p}}{v_{n o m}}}{s^{2}+k_{v} s+\frac{k_{v} k_{p}}{v_{\text {nom }}}}
$$

The result is a second order transfer function with two poles and one zero. Within control theory, a system is considered asymptotically stable if the real portion of all the poles are negative. When the poles are negative real numbers, the system response will be a damped exponential, and if the poles are complex conjugates with a negative real portion, the response will be a damped oscillation. Equation 15 can be rewritten into an augmented version of the standard canonical form that includes a zero in the numerator.

$$
\frac{E_{i-1}}{E_{i}}=\frac{\left(\frac{1}{z} s+1\right) \omega_{n}^{2}}{s^{2}+2 \zeta \omega_{n} s+\omega_{n}^{2}}
$$

Here, $\omega_{n}$ is the natural frequency, $\zeta$ is the damping ratio, and $-z$ is the zero (i.e., the value of $s$ where the numerator of equation 16 equals zero). In a standard second order system without any zeros, damping ratios greater than one will result in exponential decay, a damping ratio between zero and one will result in a damped oscillation, and a damping ratio of zero will result in a marginally stable osculation that does not grow or decay. A negative zero results in increased rise time (i.e., faster control response), and increased overshoot than the standard system.

$$
\omega_{n}=\sqrt{\frac{k_{v} k_{p}}{v_{\text {nom }}}}
$$

$$
\zeta=\sqrt{\frac{k_{v} v_{n o m}}{4 k_{p}}}
$$

$$
z=\frac{k_{p}}{k_{G S} v_{n o m}}
$$


The poles of the transfer function in equation 16 are determined by finding the values of $s$ where the denominator is equal to zero.

$$
\text { poles }=\omega_{n}\left(-\zeta \pm \sqrt{\zeta^{2}-1}\right)
$$

It can be shown that the real portion of the poles of the transfer function are less than zero if $k_{v}, k_{p}$, and $v_{n o m}$ are positive, indicating that the ASTAR-12 algorithm is string stable. Additional intuition on the effect of the gains on the string stability can be gained by examining equation 18 . Increasing values of $k_{p}$ and decreasing values of $v_{n o m}$ will result in a lower damping ratio. During an arrival, the values of $k_{p}$ increase as the aircraft approaches the achieve-by point and the nominal profile speed $\left(v_{n o m}\right)$ decreases, indicating that the ASTAR-12 algorithm will have the smallest damping ratio when the aircraft is close to the achieve-by point.

The zeros of the transfer function in equation 16 are determined by finding the values of $s$ where the numerator is equal to zero. As the zero approaches $-\infty$, the effect of the zero diminishes and the system behavior approaches the standard second order canonical form. Since the value of $v_{\text {nom }}$ is significantly larger than the value of $k_{p}$, the effect of the zero on the string stability of ASTAR-12 is small.

$$
\text { zero }=-\frac{k_{p}}{k_{G S} v_{\text {nom }}}
$$

Figure 2 shows the step response for a variety of damping ratios. Assuming that typical values of $k_{v}$ range from 0.05 to 0.2 , values of $k_{p}$ range from 0.375 to 1.5 , and values of $v_{n o m}$ range from 100 knots to 600 knots (the lowest and highest groundspeed expected by a turbojet), the range of damping ratios of the ASTAR-12 algorithm can be determined. Using this information, the damping ratio can range from a value of 0.9 to a value of 8.9 .

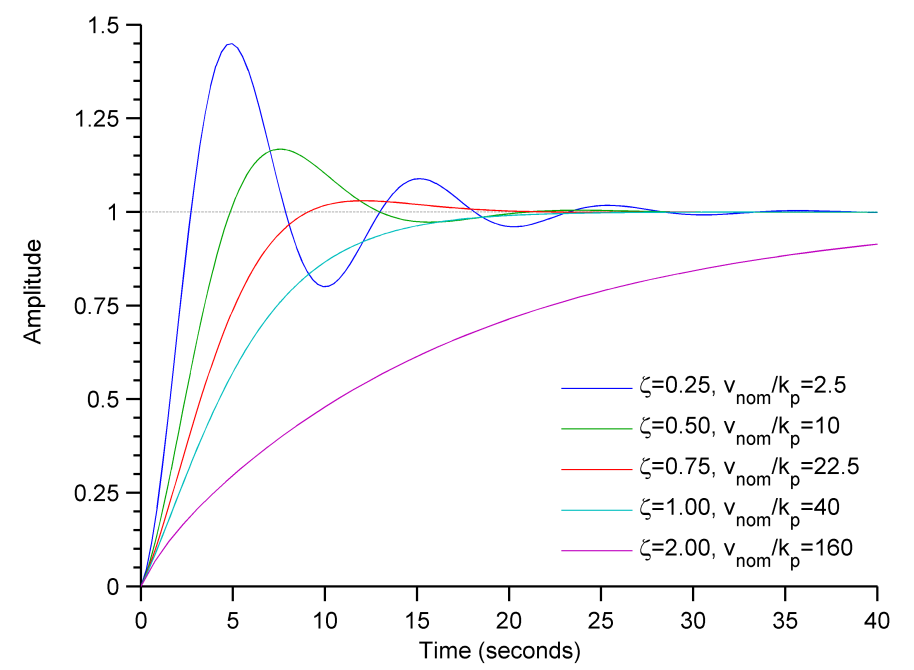

Figure 2. The step response of the error propagation function for several damping ratios $\left(k_{v}=0.1, k_{G S}=1\right.$, and $v_{n o m} / k_{p}$ is varied)

\section{Fast-Time Experiment Design}

A fast-time simulation was conducted to evaluate the performance of strings of aircraft conducting IM operations using the ASTAR-12 spacing algorithm. The simulation examined 6,720 strings of IM aircraft arriving into either Phoenix Sky Harbor airport (KPHX) or Denver International airport (KDEN). Each IM aircraft conducted IM operations from a point approximately $150 \mathrm{nmi}$ from the runway threshold to the final approach fix, which was used as the achieve-by point in this simulation. The experiment variables were selected to simulate realistic variability within the midterm national airspace system. The speed profiles of the first target aircraft (the leader of each string of IM aircraft) at KPHX were directly derived from aircraft speed profiles observed in an ATD-1 human-in-the-loop simulation and wind fields observed at 
KPHX and KDEN were used. Other aircraft parameters were randomly selected to more accurately simulate the variability expected in future IM operations in the midterm National Airspace System.

Since the objective of this simulation was to characterize the string performance of the ASTAR-12 spacing algorithm, the string position was the independent variable of interest. Three additional scenario-level variables were included in the simulation to add variability. These variables were: the speed profile flown by the first target aircraft in each string, the truth and forecast wind fields, and the destination airport (figure $3)$.

There were a total of 14 target aircraft speed profiles simulated for each airport. The goal when creating the target aircraft speed profiles was to approximate the speeds that a delayed target aircraft would fly if controlled by an air traffic controller using TMA-TM and the CMS. The target aircraft speed profiles investigated at KPHX were based off of speed profiles observed in a previous ATD-1 human-in-the-loop simulation (figure 4). To ensure that a wide range of target aircraft speed profiles were included in this simulation, the target aircraft speed profiles observed in the human-in-the-loop simulation were clustered to determine unique patterns. Twelve speed profiles were selected from the clustered dataset and combined with two nominal target aircraft speed profiles. Since human-in-the-loop simulation data was not available for KDEN, 12 off-nominal target aircraft speed profiles were created with the goal of approximating the variability of the KPHX target aircraft speed profiles. The 12 off-nominal target aircraft speed profiles were combined with two nominal speed profiles.

A total of sixteen wind cases, eight for each airport, were selected from a set of winds observed at KPHX and KDEN. Each wind case consisted of a truth wind and a forecast wind that were temporally offset in time. The ETA differences between more than a years worth of observed winds and a no wind condition were computed for the routes simulated in this experiment, and used to select a set of truth wind fields with a range of ETA differences. Wind fields within six hours of each selected truth wind were used as potential forecast wind candidates. The Root-Mean-Square (RMS) of the wind difference between each chosen truth wind and each forecast wind candidate were examined, and the forecast wind was selected to have a RMS difference of 10 to $12 \mathrm{knots}$ in the center airspace and 7 to 9 knots in the terminal airspace. To simulate the decent wind forecast that is normally available on-board the aircraft, the forecast wind field was discretely sampled using a vertical column at the runway threshold at the altitudes of 30,000 feet, 24,000 feet, 18,000 feet, 10,000 feet, and at the surface. This method of sampling the forecast winds is consistent with the capability that will likely be available in retrofit IM avionics. The discrete descent wind forecast was used by the ASTAR algorithm to predict the time-to-go of both the IM and target aircraft. The ASTAR algorithm also includes a heuristic that blends the IM aircraft's sensed winds with the wind forecast; a functionality that was used in this simulation.

In addition to the previously discussed variables, several aircraft parameters were randomly varied. To approximate conditions observed in ATD-1 human-in-the-loop simulations, the initial delay of each aircraft was selected from a normal distribution with a mean of 30 seconds and a standard deviation of 15 seconds. The aircraft dynamics model was randomly selected from a set of seven Base of Aircraft Data (BADA) ${ }^{25}$

Phoenix Sky Harbor Airport (KPHX)

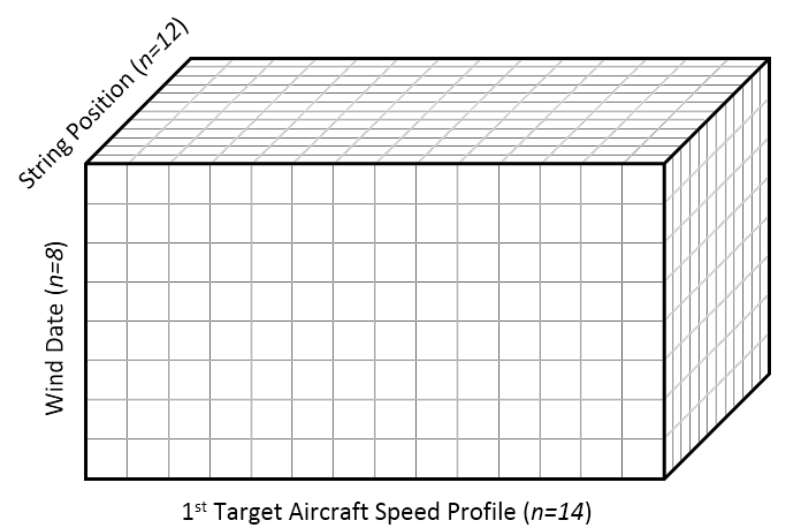

Denver International Airport (KDEN)

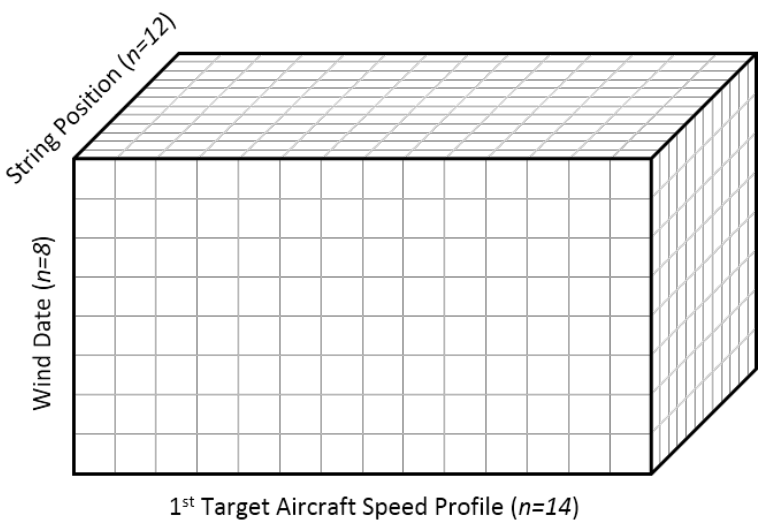

Figure 3. Experiment Design 


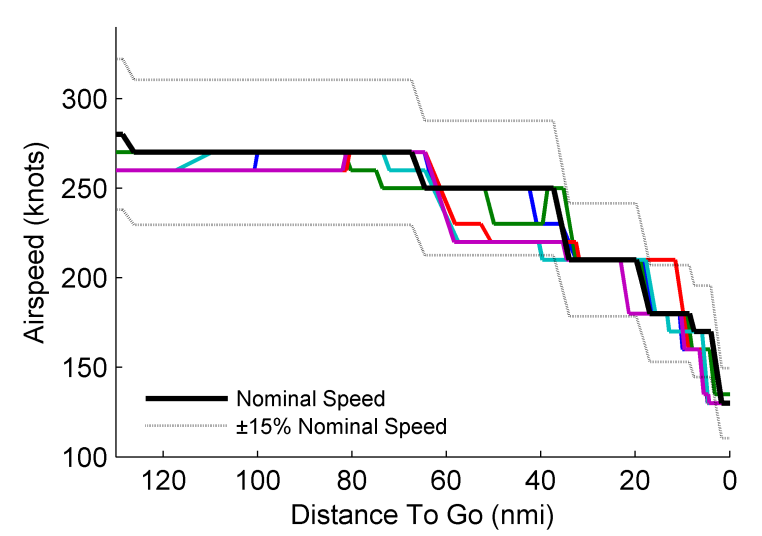

(a) East flow EAGUL arrival at KPHX

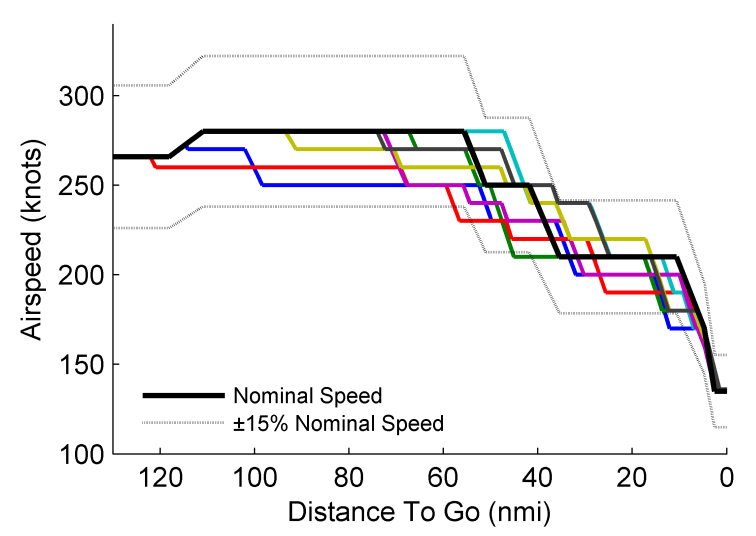

(c) North flow BOSSS arrival at KDEN

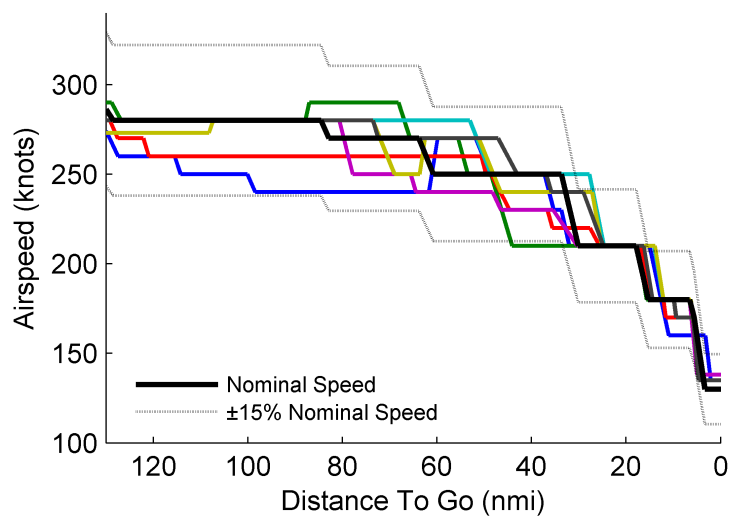

(b) East flow MAIER arrival at KPHX

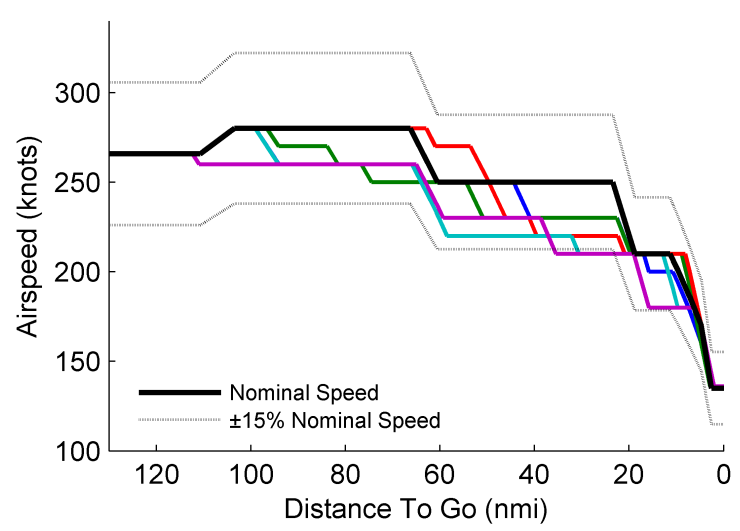

(d) North flow ANCHR arrival at KDEN

Figure 4. The target aircraft speed profile options

aircraft models using a uniform distribution. The aircraft models used in this simulation include the B737, B752, B773, A320, A319, A306, and CRJ1 models. The route of each aircraft in each string was randomly selected from a set of two routes at KPHX and two routes at KDEN using a uniform distribution, creating different merging and in-trail conditions. The route options that were simulated at KPHX were the EAGUL and MAIER arrivals in the east flow configuration to runway 08 , and the route options that were simulated at KDEN were the BOSSS and ANCHR arrivals in the north flow configuration to runway 35L. Lastly, the weight of each aircraft was randomly selected from a set of weights that ranged from the aircraft's reference weight to the aircraft's reference weight plus half of the maximum payload weight. A total of thirty replicates for each combination of independent variables were used to sample the random variables (i.e., 30 replicates of each cell in figure 3 ).

In addition to the randomized variables, a 10 second time delay was implemented to model the time it takes for pilots to recognize a new IM commanded speed and manually enter it into their aircraft. The 10 second time delay is similar to the flight crew time delay determined from previous human-in-the-loop simulations. $^{21,26}$

Aircraft state data from both the target aircraft and IM aircraft were collected and used to compute several metrics that describe the performance of the ASTAR-12 algorithm. These metrics include the delivery accuracy and precision at the achieve-by point, the schedule drift, the number of commanded speed changes, the Root-Mean-Square (RMS) of the spacing error, and the amount of speed control used. 


\section{Fast-Time Simulation Results}

\section{A. Delivery Accuracy and Precision}

The delivery accuracy and precision are measures of how accurately and precisely the ASTAR-12 algorithm achieved the desired spacing goal. The delivery accuracy was measured as the difference between the assigned spacing goal and the elapsed time between when the target and IM aircraft crossed the final approach fix. Previous experiments have shown the ability of IM algorithms to achieve precise spacing intervals with both a mean delivery accuracy and standard deviation less than five seconds. ${ }^{13,16,19,21,26}$ Overall, the mean delivery accuracy in this simulation was 2.4 seconds and standard deviation was 4.6 seconds. A total of $4.2 \%$ of the IM aircraft had delivery accuracies worse than 10 seconds.

Parsing the data by airport revealed that there was a significant difference in both the mean and standard deviation of the delivery accuracy. The mean delivery accuracy at KPHX was 2.1 seconds with a standard deviation of 3.0 seconds, and the mean delivery accuracy at KDEN was 2.7 seconds with a standard deviation of 5.7 seconds. Additionally, only $0.8 \%$ of the aircraft at KPHX had delivery accuracies worse than 10 seconds, whereas $7.5 \%$ of aircraft at KDEN had delivery accuracies worse than 10 seconds. The large discrepancy between the delivery accuracies at KPHX and KDEN is likely caused by differences in the wind conditions or the target aircraft speed profiles used in this simulation.

The delivery accuracy was also analyzed to determine if it changed as a function of string position. An Analysis of Variance (ANOVA) and Tukey multi-comparison test revealed that the delivery accuracy increased as a function of string position at both KPHX $(p<0.001)$ and KDEN $(p<0.001)$. However, the delivery accuracy results did not reveal any unstable string behavior, and the 12 th aircraft in the string had an average delivery accuracy of 3.3 seconds and a standard deviation of 3.8 seconds, which is within the acceptable range. While the delivery accuracy degraded as a function of string position, figure 5 suggests that the increase reached an asymptote at the seventh or eighth aircraft in the string.

Another observation was that the delivery accuracy had a late bias after the fourth aircraft in the string. In the next section, it will be shown that the bias in the delivery accuracy resulted in an increase in the average schedule drift as a function of string position. While there is no conclusive information on the cause of the delivery accuracy bias, the most probably cause is the lack of symmetry in the groundspeed feedback term. The groundspeed term in the ASTAR-12 algorithm is disabled when the target aircraft's groundspeed is faster than predicted to prevent undesirable speed increases from occurring. Based on the examination of case studies, random variations throughout the string can cause an IM aircraft to be commanded a speed faster then the nominal profile close to the achieve-by point. When this occurs, the groundspeed term is turned off and the ASTAR-12 algorithm reverts to proportional control. The combination of a target aircraft speed deviation and proportional control causes the ASTAR-12 algorithm to exhibit a steady-state error; potentially resulting in the delivery accuracy bias.

\section{B. Schedule Drift}

The schedule deviation, or schedule drift, was also examined as a function of string position. Large schedule drifts could cause integration problems with non-IM aircraft that are controlled to achieve a sched-

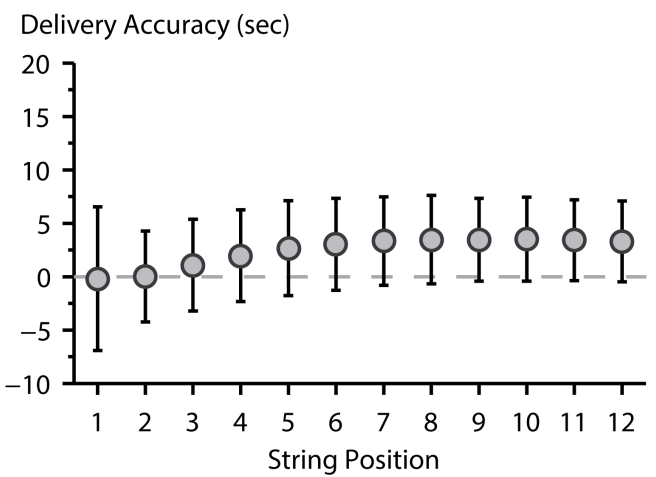

Figure 5. The delivery accuracy at the final approach fix as a function of string position (error bars represent \pm one standard deviation)

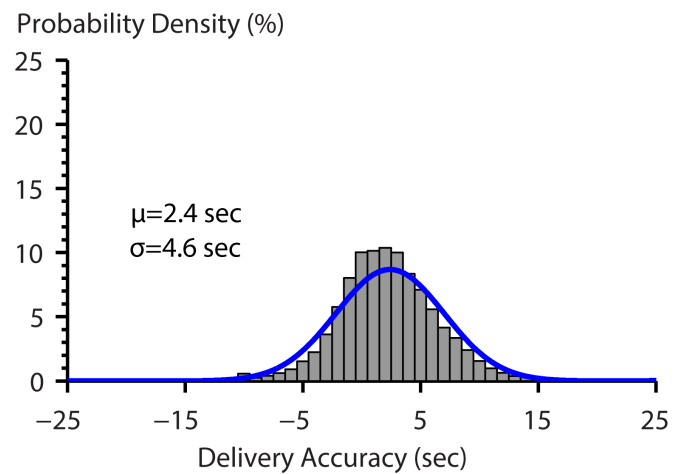

Figure 6. A histogram of the delivery accuracy at the final approach fix 


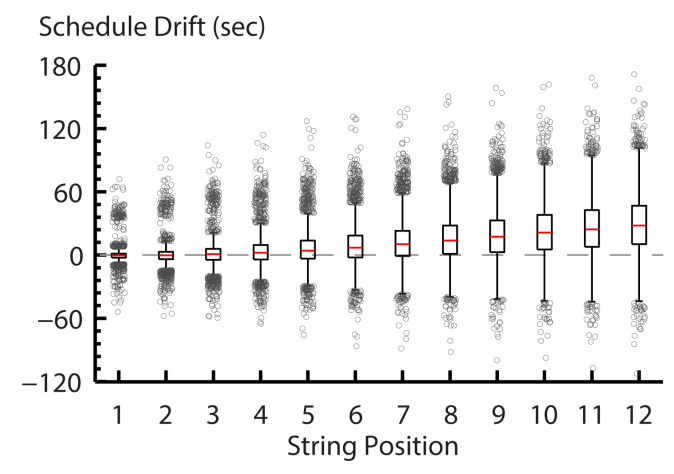

Figure 7. A boxplot of the schedule drift at the final approach fix as a function of string position

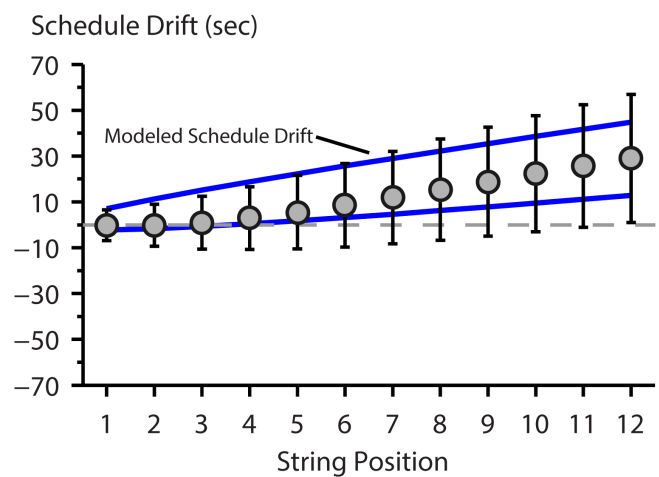

Figure 8. The modeled schedule drift at the final approach fix, adjusted for the observed schedule deviation bias, compared to the actual schedule drift (error bars represent \pm one standard deviation)

uled time of arrival. Since there were no scheduling tools modeled in this simulation, the schedule drift was computed as the sum of spacing errors of all of the aircraft in each string.

Figure 7 shows that both the mean and standard deviation of the schedule drift increased as a function of string position. The mean schedule drift increased from -0.2 seconds for the first aircraft in the string to 28.9 seconds for the last aircraft in the string. Likewise, the standard deviation of the schedule drift increased from 6.7 seconds for the first aircraft in the string to 28 seconds for the last aircraft in the string. The change in the mean schedule drift is caused by the late bias in delivery accuracy, whereas, the increase in standard deviation is an expected result based on a model described by Levitt. ${ }^{27}$

Levitt et al. derived an equation that can be used to estimate the schedule drift of a string of IM aircraft, assuming normally distributed spacing errors with zero mean. ${ }^{27}$ The equation uses the standard deviation of the delivery accuracy $\left(\sigma_{s p c}\right)$ and the standard deviation of the first target aircraft's schedule deviation $\left(\sigma_{s c h}\right)$ to model the standard deviation of the schedule deviation $\left(\sigma_{d e v}\right)$ for the $k^{t h}$ aircraft in the string.

$$
\sigma_{d e v}=\sqrt{2 \sigma_{s c h}^{2}+k \sigma_{s p c}^{2}}
$$

Using the standard deviation of the IM aircraft $(4.6 \mathrm{sec})$ and assuming that the standard deviation of the schedule deviation of the first target aircraft in the string is zero, the theoretical standard deviation of the schedule drift for the 12th aircraft in the string is 16 seconds. The observed standard deviation of the schedule drift for the 12 th aircraft in the string was 30 seconds. The theoretical calculation assumes a mean spacing error of zero and thus a mean schedule drift of zero; however, it is easy to adjust the model to include a delivery accuracy bias. The blue line in figure 8 shows \pm one standard deviation of the modeled results after they were adjusted for the observed delivery accuracy bias (i.e., 2.4 seconds was added to the mean for each string position). The larger standard deviation of the observed data is caused by the fact that the delivery accuracy was not normally distributed. Instead, several outliers conditions contributed to longer tails on the delivery accuracy distribution.

\section{Number of Speed Changes}

The number of speed changes commanded by the ASTAR-12 spacing algorithm were evaluated to ensure that they did not increase as a function of string position, which could indicate string stability problems. Figure 9 shows that the number of IM speed commands increased slightly as a function of string position; the first IM aircraft in the string had an average of 10.6 IM speed commands and the $12^{\text {th }}$ IM aircraft in the string had an average of 12.9 IM speed commands, including published speed changes. This corresponds to an average speed change rate of 0.4 speed changes per minute with a standard deviation of 0.1 speed changes per minute throughout the arrival for the first aircraft in the string, and 0.5 speed changes per minute with a standard deviation of 0.1 speed change per minute for the $12^{\text {th }}$ aircraft in the string.

A second metric that was examined was the number and magnitude of speed reversals commanded by the ASTAR-12 algorithm. The number and magnitude of speed reversals were computed by counting the number of consecutive speed increases. For instance, if there were three 10 knot speed increases in a row followed by a speed decrease, one 30 knot speed reversal was counted. Figure 10 shows the number of speed 


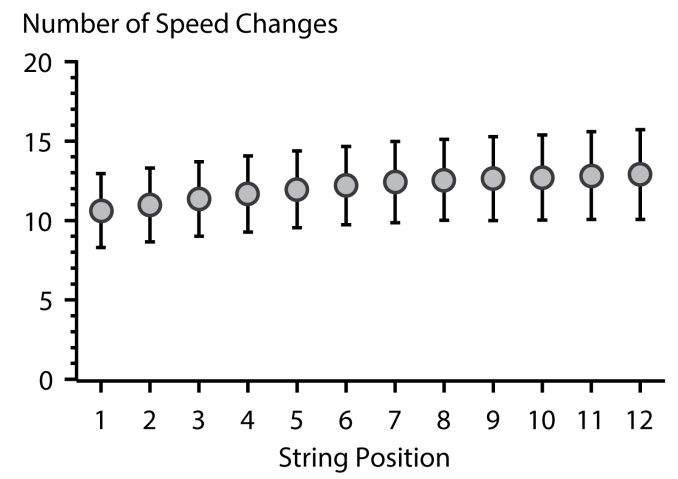

Figure 9. The number of speed changes commanded by the ASTAR-12 spacing algorithm as a function of string position (error bars represent \pm one standard deviation)
Number of Speed Reversals

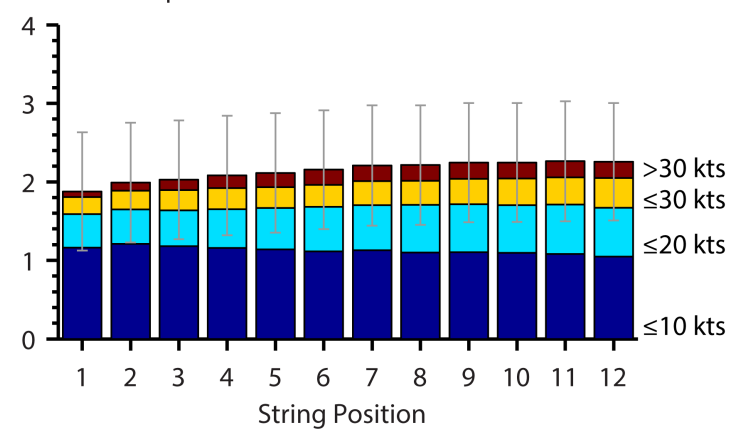

Figure 10. The number and magnitude of speed reversals commanded by ASTAR-12 as a function of string position (error bars represent \pm one standard deviation)

reversals as a function of string position, binned by the speed reversal magnitude. There was an average of 2.3 speed reversals per IM operation; however, approximately half of the speed reversals were 10 knots or less.

Figure 10 shows that the total number of speed reversals increased slightly as a function of string position. More interestingly, the average number of 10 knot speed reversals decreased as a function of string positions and the number of higher magnitude speed reversals (greater than 20 knots) increased.

\section{Root-Mean-Square of Spacing Error}

The main indication of whether an IM algorithm is string stable is whether the spacing error increases as a function of string position. The RMS of the spacing error over the entire IM operation was examined to validate the results of the analytic model presented in section III. Since each string position was provided with the same distribution of initial spacing errors (figure 13), an increase in the RMS of the spacing error as a function of string position could indicate unstable string performance. Figure 11 shows that the RMS of the spacing error did not increase as a function of string position, validating the analytic analysis conducted in section III.

\section{E. Root-Mean-Square of Speed Control}

The RMS of the speed control throughout an arrival was examined to determine if the total amount of speed control required to achieve the assigned spacing goal increased as a function of string position. Since each string position was provided with the same distribution of initial spacing errors (figure 13), an increase in the total amount of speed control as a function of string position could indicate undesirable string performance. An Analysis of Variance (ANOVA) and Tukey multi-comparison test revealed that there was a substantial increase in the total amount of speed control as a function of string position at KPHX $(p<0.001)$ and at KDEN $(p<0.001)$. Figure 12 shows that the average RMS of speed control for the first IM aircraft in the string was 11.4 knots and increased to 17.9 knots for the 12 th aircraft in the string. The increase in the amount of speed control required is likely the driver behind the small increase in the number of speed changes and the increase in the number and magnitude of speed reversals. This data suggests that the speed commands of aircraft at the end of a very long string of IM aircraft will be less desirable than the commands for the first aircraft in the string.

\section{Conclusions}

Both an analytic analysis and a fast-time simulation were conducted to characterize the string performance of the ASTAR-12 spacing algorithm. The analytic analysis included the derivation of an error propagation transfer function. Both the poles of the transfer function and the damping ratio indicated that the ASTAR-12 spacing algorithm is string stable. The fast-time simulations examined 6,720 strings of IM aircraft arriving into either KPHX or KDEN. The independent variables that were examined were the destination airport, string position, wind field, and the speed profile of the first target aircraft in each string. 


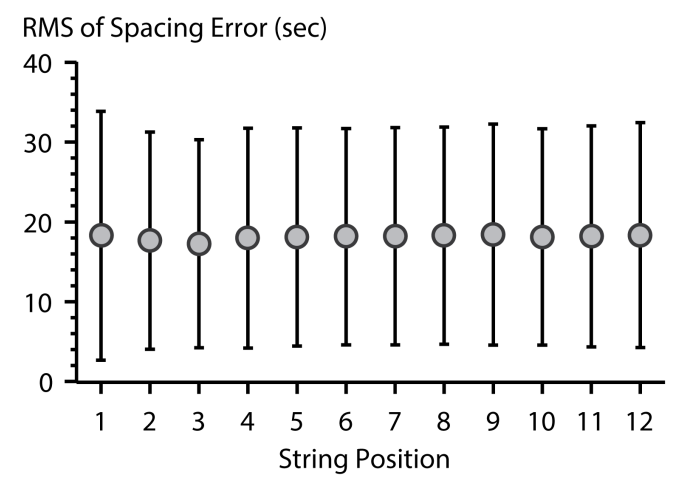

Figure 11. The Root-Mean-Square (RMS) of the spacing error as a function of string position (error bars represent \pm one standard deviation)

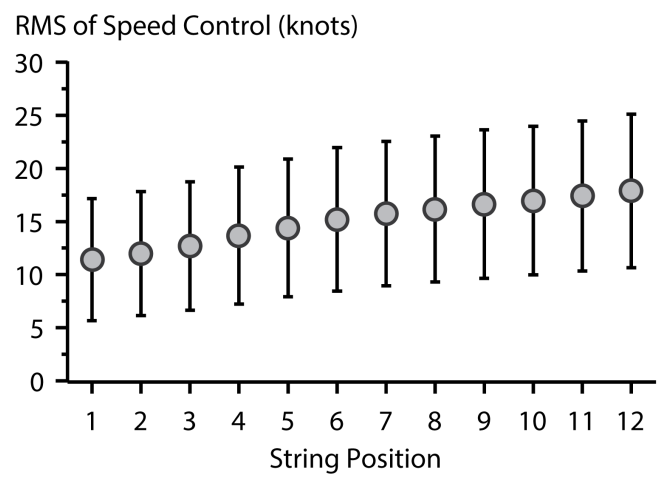

Figure 12. The Root-Mean-Square (RMS) of the speed control used to achieve the spacing goal as a function of string position (error bars represent \pm one standard deviation)

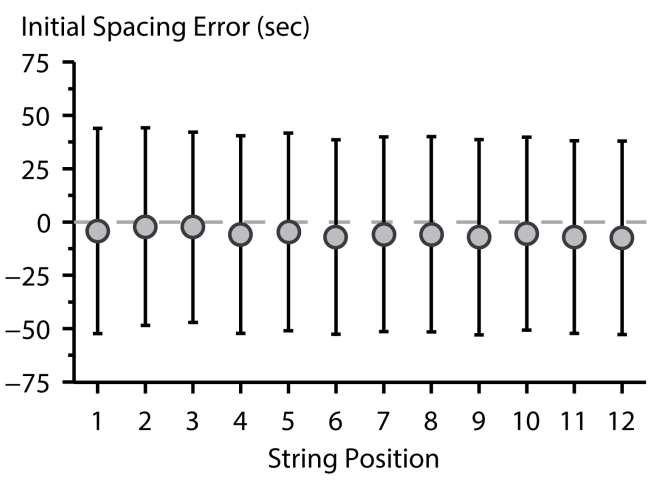

Figure 13. The initial spacing error for each aircraft in the string was sampled from the same distribution (error bars represent \pm one standard deviation)

Additional aircraft parameters were randomly varied to better simulate the variability expected in future IM operations. The data was examined to characterize the string performance of the ASTAR-12 spacing algorithm. The results did reveal degradation of ASTAR-12 performance as a function of string position; however the performance is still in the acceptable range for strings of moderate length.

\section{References}

${ }^{1}$ Babbitt, R., "FAA Aerospace Forecast: Fiscal Years 2011-2031," US Department of Transportation Federal Aviation Administration Aviation Policy and Plans, 2010, pp. 2011-2031.

${ }^{2}$ Robinson, J. and Kamgarpour, M., "Benefits of continuous descent operations in high-density terminal airspace under scheduling constraints," 10th AIAA Aviation Technology, Integration, and Operations (ATIO) Conference, Fort Worth, TX, 2010, pp. 13-15.

${ }^{3}$ Baxley, B., Swenson, H., Prevot, T., and Callantine, T., "NASA's ATM technology demonstration-1: Integrated Concept of arrival operations," Digital Avionics Systems Conference (DASC), 2012 IEEE/AIAA 31st, IEEE, 2012, pp. 3B1-1.

${ }^{4}$ Isaacson, D. R., Robinson III, J. E., Swenson, H., and Denery, D., "A concept for robust, high density terminal air traffic operations," 10th AIAA Aviation Technology, Integration, and Operations (ATIO) Conference, Fort Worth, TX, 2010.

${ }^{5}$ Swenson, H. N., Thipphavong, J., Sadovsky, A., Chen, L., Sullivan, C., and Martin, L., "Design and evaluation of the terminal area precision scheduling and spacing system," 2011.

${ }^{6}$ Callantine, T., Palmer, E., Kupfer, M., et al., "Human-in-the-loop simulation of trajectory-based terminal-area operations," 27th International Congress of the Aeronautical Sciences (ICAS), Nice, France, 2010.

${ }^{7}$ Kupfer, M., Callantine, T., Martin, L., Mercer, J., and Palmer, E., "Controller support tools for schedule-based terminalarea operations," Proceedings of the Ninth USA/Europe Air Traffic Management Research and Development Seminar, 2011.

${ }^{8}$ Barmore, B. E., Abbott, T. S., Capron, W. R., and Baxley, B. T., "Simulation results for airborne precision spacing along continuous descent arrivals," perspective, Vol. 22, 2008, pp. 27.

${ }^{9}$ Murdoch, J. L., Barmore, B. E., Baxley, B. T., Capron, W. R., and Abbott, T. S., "Evaluation of an airborne spacing concept to support continuous descent arrival operations," 2009.

${ }^{10}$ Abbott, T., "An Overview of a Trajectory-Based Solution for En Route and Terminal Area Self-Spacing to Include Parallel Runway Operations," Nasa/cr-2011-217194, 2011. 
${ }^{11}$ Abbott, T., "An Overview of a Trajectory-Based Solution for En Route and Terminal Area Self-Spacing: Fourth Revision," Nasa/cr-2013-218044, 2013.

${ }^{12}$ Barmore, B., Abbott, T., and Krishnamurthy, K., "Airborne-managed spacing in multiple arrival streams," Proceedings of the 24th International Congress of the Aeronautical Sciences, Yokohama, Japan, 2004.

${ }^{13}$ Barmore, B. E., Abbott, T. S., and Capron, W. R., "Evaluation of airborne precision spacing in a human-in-the-loop experiment," AIAA 5th Aviation Technology, Integration, and Operations Conference (ATIO), 2005, pp. 26-28.

${ }^{14}$ Barmore, B., "Airborne Precision Spacing: A Trajectory-Based Aprroach to Improve Terminal Area Operations," 25th Digital Avionics Systems Conference, 2006 IEEE/AIAA, IEEE, 2006, pp. 1-12.

${ }^{15}$ Murdoch, J., Wilson, S., Hubbs, C., and Smail, J., "Acceptability of Flight Deck-Based Interval Management Crew Procedures," August 19-22 2013.

${ }^{16}$ Wilson, S., Murdoch, J., Hubbs, C., and Swieringa, K., "Evaluation of Flight Deck-Based Interval Management Crew Procedure Feasibility," August 19-22 2013.

${ }^{17}$ Swieringa, K., Murdoch, J., Baxley, B., and Hubbs, C., "Evaluation of an Airborne Spacing Concept, On-board Spacing Tool, and Pilot Interface," September 20-22 2011.

${ }^{18}$ Baxley, B., Swieringa, K., and Capron, W., "Interval Management with Spacing to Parallel Dependent Runways (IMSPiDR) Experiment and Results," 28th International Congress of the Aeronautical Sciences (ICAS2012), 2012.

${ }^{19}$ Swieringa, K. A., Underwood, M. C., Barmore, B., and Leonard, R. D., "An Evaluation of a Flight Deck Interval Management Algorithm including Delayed Target Trajectories," June 16-20 2014.

${ }^{20}$ Abbott, T. S., "A Brief History of Airborne Self-Spacing Concepts," Tech. rep., 2009.

${ }^{21}$ Baxley, B., Murdoch, J., Swieringa, K., Barmore, B., Capron, W., Hubbs, C., Shay, R., and Abbott, T., "Experiment Description and Results for Arrival Operations Using Interval Management with Spacing to Parallel Dependent Runways (IMSPiDR)," 2013.

${ }^{22}$ Baxley, B., Johnson, W., Swenson, H., Robinson, J., Prevot, T., Callantine, T., Scardina, J., and Greene, M., "Air Traffic Management Technology Demonstration-1 Concept of Operations (ATD-1 ConOps)," Nasa/tm-2012-217585, Aug 2012.

${ }^{23}$ Weitz, L. and Hurtado, J., "String Stability Analysis of Selected Speed Control Laws for Interval Management," AIAA Guidance, Navigation, and Control Conference, 2012.

${ }^{24}$ Krishnamurthy, K., Barmore, B., Bussink, F., Weitz, L., and Dahlene, L., "Fast-time evaluations of airborne merging and spacing in terminal arrival operations," AIAA Guidance, Navigation, and Control Conference and Exhibit, 2005.

${ }^{25}$ Center, E. E., "User Manual for the Base of Aircraft Data (BADA), Revision 3.6," EEC note, , No. 10/04, 2004.

${ }^{26}$ Swieringa, K. A., Wilson, S. R., and Shay, R., "An Evaluation of Retrofit Flight Deck Displays for Interval Management," June 16-20 2014.

${ }^{27}$ Levitt, I. M., Weitz, L. A., Barmore, B. E., and Castle, M. W., "Modeling Delay and Delivery Accuracy for Mixed Absolute and Relative Spacing Operations," 14th AIAA Aviation Technology, Integration, and Operations Conference, Atlanta, GA, 16-20 June 2014. 